\title{
Invaginación yeyunal por metástasis intestinal de un carcinoma primario pulmonar: aportación de un caso y revisión de la literatura
}

\author{
M. J. Oruezábal Moreno ${ }^{1}$, M. R. Burón Fernández ${ }^{2}$, R. Lara Lopez-Dóriga ${ }^{1}$, R. Espinosa Aunión ${ }^{1}$
}

\section{Resumen}

Las metástasis intestinales por cáncer de pulmón, son entidades clínicas muy raras, que excepcionalmente, se manifiestan antes que el tumor primario. El diagnóstico se basa en la expresión inmunohistoquímica de diversos tipos de queratinas y marcadores específicos pulmonares como TTF-1. El tratamiento se enfocará en el contexto de un cáncer de pulmón metastásico, aunque puede requerirse una resección quirúrgica intestinal en caso de perforación, hemorragia u obstrucción intestinal, como el presente caso.

La supervivencia, en los pocos casos publicados es escasa, no superando las 20 semanas, a pesar del tratamiento realizado.

Presentamos un caso de una invaginación intestinal por metástasis de un carcinoma anaplásico de pulmón.

Palabras clave: Metástasis intestinal. Invaginación intestinal. TTF-1.

Oncología, 2006; 29 (9):387-391

\section{Summary}

Intestinal metastases from primary lung carcinoma are of rare presentation, and exceptionally, can appear before the primary tumor manifestations. The diagnosis is based on the expression of some cytokeratins and specific lung markers, as TTF-1. Obviously, although focusing on the treatment of the metastatic lung cancer, a surgical intestinal resection in case of perforation, intestinal hemorrhage or obstruction is mandatory, as it happened in the case we report. According to the few published cases, survival is poor, not surpassing 20 weeks in spite of treatment. We present a case of intestinal intussusception caused by metastases from a primary anaplastic carcinoma of the lung.

Key words: Intestinal metastasis. Intestinal intussusception. Cytokeratins. TTF-1.

1 Sección Oncología Médica

2 Servicio de Medicina Interna 


\section{Introducción}

Las metástasis intestinales por cáncer de pulmón son excepcionales y, en la mayoría de los casos, se diagnostican con posterioridad a la neoplasia pulmonar.

Es evidente que en las metástasis, el diagnóstico preciso del tumor primario o incluso diferenciarlo de un tumor metastático, puede ser difícil. Además el cáncer de pulmón puede infiltrar la pleura y simular un mesotelioma maligno, o incluso que ambas neoplasias se originen simultáneamente. Por todo ello, la identificación del origen de las metástasis ha sido objeto de especial atención y existen numerosos estudios que han aplicado la inmunohistoquímica para precisar el diagnóstico. Uno de los principales avances en este campo, ha sido el uso de queratinas de diferente peso molecular para diagnosticar distintos tipos de carcinomas, y la identificación de marcadores específicos como Antígeno Prostático Específico, Receptores de Estrógenos y de Progesterona, Antígeno Carcinoembrionario (CEA), Proteína S100, Calretinina, HEP, Tiroglobulina y TTF-1, capaz, esta última, de inmunomarcar los núcleos de al menos el 76\% de los casos de cáncer de pulmón.

El caso que presentamos se manifestó inicialmente con clínica digestiva que llevo posteriormente al diagnóstico de invaginación intestinal por una metástasis de carcinoma anaplásico pulmonar, diagnóstico establecido gracias al patrón de expresión inmunohistoquímica.

\section{Caso clínico}

Varón de 43 años, fumador activo de unos 15 cigarrillos al día. Acudió a Urgencias por presentar dolor epigástrico continuo de comienzo brusco acompañado de náuseas y mareo. Hábito intestinal normal. Refiere episodios similares de menor intensidad que relaciona con la ingesta y que cedían con el ayuno. Astenia y anorexia moderada, con pérdida de peso de unos $15 \mathrm{Kg}$ en los tres últimos meses. A la exploración estaba afebril, TA 80/40, palidez cutáneo-mucosa y el abdomen era blando, depresible, sin defensa ni signos de irritación peritoneal, aunque doloroso a la palpación en epigastrio con peristaltismo discretamente aumentado. En la analítica destacó un $\mathrm{Ht}^{\circ}$ de $30 \%$ con una $\mathrm{Hb}$ de $11.1 \mathrm{~g} / \mathrm{dl}$ siendo el resto normal.

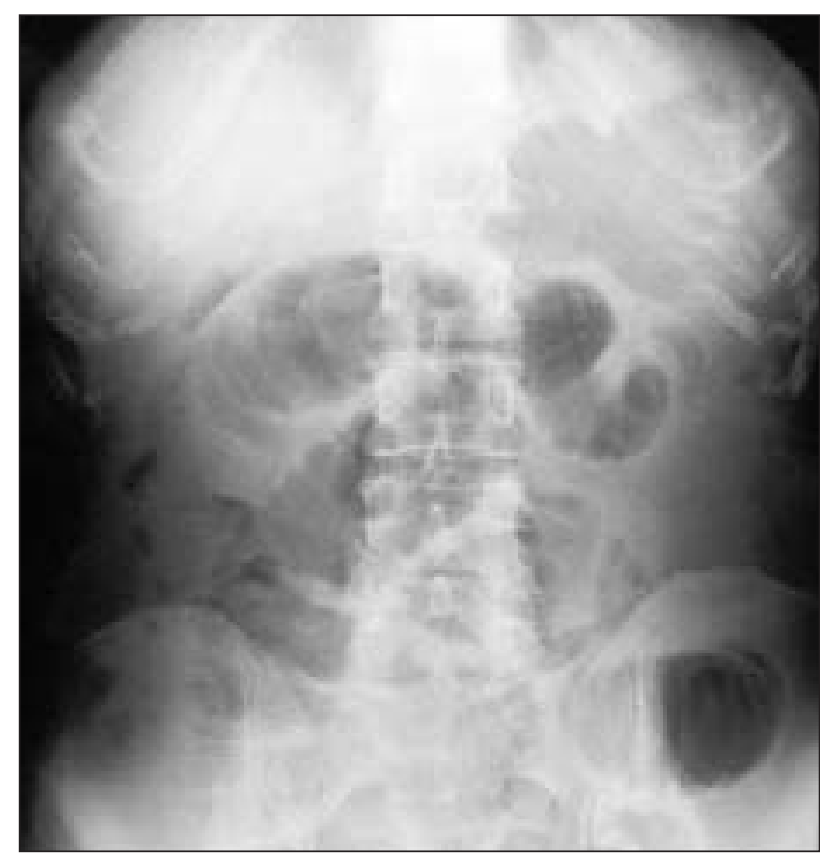

Figura 1. Radiografía de abdomen. Dilatación de asas yeyunales.

La radiografía de abdomen se informó como dilatación de asas yeyunales con niveles hidroaereos (Figura 1). La ecografía abdominal puso de manifiesto la presencia en el plano de corte sagital una imagen en "pseudoriñón o sandwich" y en el corte axial imagen en "diana, ojo de buey o donnuts". La capa interna era hiperecoica y la capa externa hipoecoica, sospechoso de invaginación intestinal (Figura 2). Se decidió intervención quirúrgica y tras la inducción

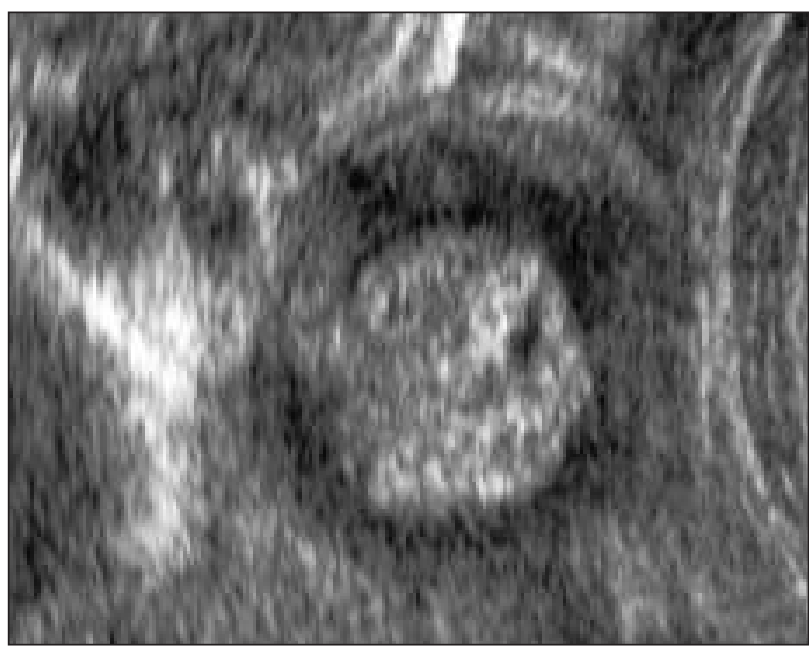

Figura 2. Ecografía abdominal. Imagen en "diana, ojo de buey o donnuts". La capa interna era hiperecoica y la capa externa hipoecoica. 


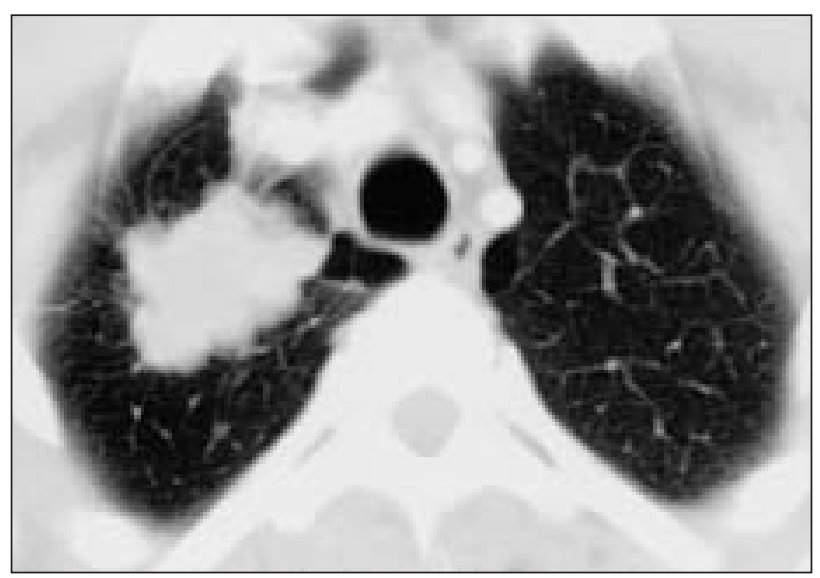

Figura 3. TC torax. Masa hiliar derecha de unos $4 \mathrm{~cm}$, polilobulada y de contornos espiculados, que engloba el bronquio lobar superior.

anestésica se palpó una masa abdominal localizada en mesogastrio lisa y móvil. Se realizó una laparotomía supraumbilical, apreciandose una intususpección de un asa yeyunal que fue imposible de reducir, por lo que se procedió a resección segmentaria de $30 \mathrm{~cm}$ de yeyuno conteniendo una tumoración de 4 x 4 x 2,5 $\mathrm{cm}$ que se informó como carcinoma anaplásico, con fenotipo rabdoide que invadía toda la pared e infiltraba la capa subserosa. El estudio inmunohistoquímico mostró alta positividad para citoqueratinas AE-1/AE3 , citoqueratina 7 , citoqueratinas $5 / 6 / 8 / 18$ y vimentina. Fueron negativos la citoqueratina 20, y los marcadores CD-30, CD-31, actina, desmina, CD-117, MELAN-A/M arp-1 y TTF-1, lo cual orientaba a un origen pulmonar.

La TC torax confirmó la presencia de una masa hiliar derecha de unos $4 \mathrm{~cm}$, polilobulada y de contornos espiculados, que englobaba el bronquio lobar superior aunque sin estenosarlo significativamente (Figura 3). Se realizó un broncoscopia que destacó un engrosamiento de la carina en su separación con el bronquio lobar superior derecho, con tumoración endobronquial que impedía el paso del broncoscopio al segmento posterior. La biopsia confirmó el diagnóstico de un carcinoma indiferenciado con el mismo patrón inmunohistoquímico que la masa intestinal.

El postoperatorio cursó sin complicaciones, siendo dada de alta en buena situación clínica. Posteriormente fue tratado con carboplatino AUC 5 día 1, y gemcitabina $1250 \mathrm{mg} / \mathrm{m}^{2} /$ día dias 1 y 8 , cada 21 días, con respuesta parcial tras el cuarto ciclo de quimioterapia. A los seis meses del diagnóstico ingreso por deterioro cognitivo progresivo secundario a múltiples metástasis cerebrales frontoparietales derechas, falleciendo quince días después.

\section{Discusión}

Las neoplasias malignas del intestino delgado son raras, estimando su incidencia entre el 1 y $6 \%$ del total de neoplasias gastrointestinales ${ }^{1}$. La mayoría son tumores primarios malignos, como tumores del estroma gastrointestinal (GIST), y son infrecuentes los tumores metastásicos (1-4\% en las series post mortem $)^{2}$. Los tumores que metastatizan con mayor frecuencia en el intestino delgado son el melanoma maligno, el cáncer de pulmón y el de colon. Otros que lo hacen, pero con menor frecuencia, son el cáncer de mama, riñón, cervix, osteosarcoma y ovario $^{3-7}$. Generalmente, no son la primera manifestación de la diseminación tumoral y se asocian a metástasis en otros órganos.

Las metástasis intestinales por cáncer de pulmón, son entidades clínicas muy raras. Antler y cols. afirman que el cáncer de pulmón indiferenciado de células grandes y de células pequeñas produce metástasis más frecuentemente que otros tipos histológicos; mientras que McNeill y cols. y Mossier DM y cols. afirman que las metástasis son más frecuentes en los carcinomas escamosos ${ }^{9-11}$. Excepcionalmente, las metástasis intestinales se manifiestan previamente al tumor primario. La localización más frecuente suele ser el yeyuno, íleon o ambas y, menos en el duodeno ${ }^{12}$. Inicialmente se presentan por dolor abdominal, secundario a perforación u obstrucción intestinal, o hemorragia digestiva. En nuestro caso, la lesión pulmonar se diagnosticó después de los sintomas digestivos debido a una obstrucción intestinal por una invaginación yeyuno-yeyunal, entidad rara, que supone el 5\% de todas las causas de obstrucción y generalmente se asocia a la presencia de un tumor que actúa como cabeza de la intususpección ${ }^{13}$.

El diagnóstico habitualmente se realiza tras estudio baritado gastrointestinal, ecografía y TC que demuestran la presencia de una tumoración de intestino delgado con signos radiológicos de intususpección.

El tránsito intestinal baritado pondrá de manifiesto imágenes de defectos de repleción en los tumores expansivos, mientras que en los tumores invasivos 
mostrará múltiples alteraciones radiológicas (estenosis con dilatación proximal, engrosamiento de pliegues, rigidez de un segmento intestinal, ulceración o masas polipoideas múltiples).

La TC abdominal permite demostrar tres patrones diferentes dependiendo del plano de sección y la severidad del cuadro. La imagen en "diana" aparece en la fase precoz en el corte transversal, imagen en forma de "salchicha" en el corte longitudinal o "pseudo riñón” en la fase más severa debido al edema y compromiso vascular con engrosamiento de la pared intestinal.

La ecografía abdominal es la técnica de mayor rendimiento con un $100 \%$ de sensibilidad y un 88 93\% de especificidad. Presenta en el plano de corte sagital imagen en "pseudoriñón o sandwich" y en el corte axial imagen en "diana, ojo de buey o donnuts". La capa interna es hiperecoica (asa invaginada rodeada de mesenterio) y la capa externa es hipoecoica (asa donde se ha producido la invaginación), también pueden aparecer múltiples capas de ecogenicidad alternante.

El tratamiento de la intususpección es la resección segmentaria del intestino afecto sobre todo teniendo en cuenta que la cabeza de la invaginación suele ser un tumor cuyo origen es imposible de determinar intraoperatoriamente, aunque tumores obviamente malignos son tratados con amplia resección intestinal que incluirá el mesenterio adyacente con los ganglios regionales. La quimioterapia dependerá de las características del tumor y el estado del paciente, aunque según la mayoría de los casos publicados el pronóstico es ominoso ${ }^{14-20}$.

El diagnóstico definitivo se establecerá tras la cirugía siendo la inmunohistoquímica fundamental en los tumores metastásicos, ya que esclarecerá el diagnóstico diferencial planteado por el patólogo cuando se enfrenta a la histopatología de un tumor con criterios de malignidad. Durante los últimos años han surgido algunos intentos de confeccionar paneles de anticuerpos que faciliten una primera orientación ante un paciente con cáncer metastásico de origen desconocido. La expresión inmunohistoquímica de diversos tipos de queratinas en los epitelios simples, queratinizados y no queratinizados, sirve para diferenciar entre los tumores epiteliales de los no epiteliales, y lograr un diagnóstico más certero de las metástasis en las neoplasias epiteliales. La American Society Clinical Oncology, reco- mendó en el año 2002 un estudio inmunohistoquímico progresivo en función de los resultados obtenidos con una inmunotinción inicial, como punto de partida, con una mezcla de anticuerpos monoclonales contra diversas citoqueratinas, que incluyeran CK20, CK7, AE1/AE3, CAM 5.2, MAK-6 y Ker20 , y dirigir el resto de marcadores inmunohistoquímicos a partir de aquí ${ }^{21,22}$.

La citoqueratina 20 (CK-20) es un anticuerpo que ha probado utilidad en el diagnóstico de carcinoma de células de Merkel de piel, tumores de vías gastrointestinales, neoplasias mucinosas de ovario y carcinomas de células transicionales. Por su parte la queratina 7 (CK-7) es positiva mas bien en adenocarcinomas que no son de las vías gastrointestinales, como en los de mama y pulmón. Por estas características generales, el tipo de tinción inmunohistoqímica de CK-7 y CK-20 puede ser útil en el diagnóstico diferencial de carcinoma metastático de origen desconocido.

El anticuerpo AE1/AE3, es una mezcla de queratinas de bajo y de alto peso molecular, que reacciona con epidermis corneal, epitelio escamoso estratificado de órganos internos, epitelio estratificado, queratinocitos hiperproliferados y epitelios simples. EMA es una glucoproteina situada dentro de la membrana apical de células glandulares, identificandola en innumerables carcinomas epiteliales como los de mama, pulmón, estómago, intestino, próstata, riñón, tiroides y piel. El uso de EMA y de la queratina AE1/AE3 son de utilidad para precisar el diagnóstico del carcinoma escamoso de pulmón.

Actualmente disponemos de algunos marcadores inmunohistoquímicos realmente específicos y claramente relacionados con el tumor primario que facilitan una primera orientación ante un paciente con cáncer metastásico de origen desconocido. TTF-1 es una proteína de transcripción nuclear expresada por el gen de la familia NKx 2, la cual se expresa selectivamente en las células del diencéfalo embrionario y en las células embrionarias y maduras del pulmón y de la glándula tiroides ${ }^{23}$. El Factor 1 de Transcripción Nuclear Tiroideo ha resultado bastante específico para ser utilizado en el inmunomarcaje de los tumores de origen pulmonar, especialmente en los adenocarcinomas no mucinosos y en los tumores de células redondas. La expresión de TTF-1 en las neoplasias pulmonares varía en los distintos tipos histológicos. La mayoría de adenocarcinomas pulmona- 
res son positivos, mientras que, los carcinomas pobremente diferenciados, escamosos o adenocarcinomas mucinosos pueden ser negativo. Los tumores neuroendocrinos de origen pulmonar expresan TTF1 a diferencia del tumor de Merkel que es negativo y de otros tumores neuroendocrinos extrapulmonares que lo expresan con menor frecuencia ${ }^{24}$.

En el caso presentado, la combinación de queratina 7 y 20, con otros anticuerpos como AE1/AE3 y citoqueratinas $5 / 6 / 8 / 18$, sirvieron para establecer un origen pulmonar a pesar de la negatividad a TTF-1, esperable por el diagnóstico de carcinoma pobremente diferenciado.

\author{
Correspondencia: \\ Dr. M. J. Oruezábal Moreno \\ Sección Oncología Médica \\ Hospital General La Mancha Centro \\ C/ Constitución, 3 \\ E-13600 Alcázar de San Juan (Ciudad Real) \\ mauroo@sescam.jccm.es
}

\section{Bibliografía}

1. Gill SS, Heuman DM, et al. Small intestinal neoplasms. J Clin Gastroenterol 2001; 33 (4): 267-282.

2. Washington K, McDonagh D. Secondary tumors of gastrointestinal tract: surgical pathologic findings and comparison with autopsy survey. Mod Pathol 1995; 8 (4): 427233.

3. Jarry O, Vincent M, van Straaten L, Girodet B, Laennec E. Intestinal metastasis of pulmonary cancers. Apropos of 2 cases. Rev Pneumol Clin.1990; 46(6):283-286.

4. Hung GY, Chiou T, Hsieh YL, Yang MH, Chen WY . Intestinal metastasis causing intussusception in a patient treated for osteosarcoma with history of multiple metastases: a case report. Jpn J Clin Oncol. 2001; 31(4):165-167.

5. Chandramohan K, Somanathan T, Kusumakumary P, Balagopal PG, Pandey M. Metastatic osteosarcoma causing intussusception. J Pediatr Surg. 2003; 38(10):E1-3.

6. Testini M, Trabucco S, Di Venere B, Piscitelli D. Ileal intussusception due to intestinal metastases from primary malignant melanoma of the lung. Am Surg. 2002; 68(4):377-379.

7. Nozawa H, Tsuchiya M, Kobayashi T, Morita H, Kobayashi I, Sakaguchi M, et al. Small intestinal metastasis from renal cell carcinoma exhibiting rare findings. Int J Clin Pract. 2003;57(4):329-331.

8. Antler As, Ough Y, et al. Gastrointestinal metastasis from malignant tumors of the lung. Cancer 1982; 49: 170-172.

9. McNeill PM, Lawrence D, et al. Small bowel metastasis from primary carcinoma of the lung. Cancer 1987; 59: 1486-1489.

10. Mossier DM, Bloch RS, et al. Small bowel metastases from primary lung carcinoma. Am Surg 1992; 58 (11): 677-682.

11. Berger A, Cellier C, et al. Small bowel metastases from primary carcinoma of the lung. Am J Gastroenterol 1999; 94 (7): 1884-1887.

12. Kadakia SC, Parker A, et al. Metastatic tumors to the upper gastrointestinal tract: endoscopic experience. Am J Gastroenterol 1992; 87 (10): 1418-1423.

13. Martín JG, Aguayo JL, Aguilar J, Torralba JA, Lion R, Miguel J et al. Invaginación intestinal en el adulto. Presentación de 7 casos con énfasis en el diagnóstico preoperatorio. Cir Española 2001; 69: 93-97.

14. Savanis G, Simatos G, Lekka I, Ammari S, Tsikkinis C, Mylonas A, Kafasis E, Nissiotis A. Abdominal metastases from lung cancer resulting in small bowel perforation: report of three cases. Tumori. 2006;92(2):185-187.

15. Tomas D, Ledinsky M, Belicza M, Kruslin B. Multiple metastases to the small bowel from large cell bronchial carcinomas. World J Gastroenterol. 2005;11(9):1399-1402.

16. Hillenbrand A, Strater J, Henne-Bruns D. Frequency, symptoms and outcome of intestinal metastases of bronchopulmonary cancer. Case report and review of the literature. Int Semin Surg Oncol. 2005;2:13

17. Ogiso S, Maeno A, Nagahama K, Nakamura K, Okuno H. Small intestinal metastases from renal cell carcinoma: a case report and literature review. Hinyokika Kiyo. 2005;51(1):13-16..

18. Garwood RA, Sawyer MD, Ledesma EJ, Foley E, Claridge JA. A case and review of bowel perforation secondary to metastatic lung cancer. Am Surg. 2005;71(2):110-116.

19. Nozawa H, Tsuchiya M, Kobayashi T, Morita H, Kobayashi I, Sakaguchi M, Mizutani T, Tajima A, Kishida Y, Yakumaru K, Kagami H, Sekikawa T.Small intestinal metastasis from renal cell carcinoma exhibiting rare findings. Int J Clin Pract. 2003;57(4):329-331.

20. Renault PA, Arotcarena R, Cales V, Lippa A, Benichou M, Laurent P, Laborde Y. Metastatic obstruction of the small bowel revealing or complicating squamous-cell lung cancer. Two cases and a review of the literature. Rev Pneumol Clin. 2003;59(3):161-165.

21. DeYoung BR, Wick MR. Immunohistologic evaluation of metastatic carcinomas of unknown origin: an algorithmic approach. Semin Diagn Pathol 2000; 17:184-193.

22. Netto GJ. Logical approach to the Unknown Primary Cancer. Tumor panel sessions. Am Soc Clin Oncol 2002.

23. Ordoñez NG. Value of Thyroid Transcription Factor-1 immunostaining in distinghising Small Cell Lung carcinomas from other Small Cell carcinomas. Am J Surg Pathol 2000, 24:1217-1223.

24. García Tamayo J, Hernández C. Algunas consideraciones sobre la inmunohistoquímica en el diagnóstico diferencial entre Mesotelioma y Adenocarcinoma. Vitae Academia Biomédica Digital, 2002, Vol 13. 